\title{
Repair of upper eyelid retraction: a comparison between adjustable and non-adjustable sutures
}

\author{
Susan M Tucker, Richard Collin
}

\begin{abstract}
Background-The challenge with the surgical repair of upper eyelid retraction is to obtain a more predictable and satisfactory postoperative eyelid contour and height, and to minimise the need for further surgery.

Methods-A retrospective analysis was performed on all patients treated surgically for eyelid retraction at Moorfields Eye Hospital over a 13 year period.

Results-In this study good postoperative results were obtained in 10 of $13(77 \%)$ eyelids using adjustable sutures, compared with 56 of $148(38 \%)$ eyelids using non-adjustable sutures $(\mathbf{p}<0 \cdot 01)$. With non-adjustable sutures, good results were achieved more often for non-thyroid compared with thyroid related eyelid retraction $(26 / 55$ versus $31 / 93$ eyelids respectively, $\mathbf{p}<0.05)$. Previous eyelid surgery did not affect outcome.

Conclusion-We recommend the use of adjustable sutures for the repair of upper eyelid retraction, particularly in patients with thyroid disease.
\end{abstract}

(Br f Ophthalmol 1995; 79: 658-660)

Upper eyelid retraction is difficult to treat surgically, as postoperative eyelid position is highly unpredictable, particularly in patients with thyroid disease. Numerous surgical procedures have been devised ${ }^{1-6}$ in an attempt to improve postoperative results. Although the adjustable suture technique has recently been reported in the repair of ptosis ${ }^{7-9}$ and upper eyelid retraction, ${ }^{10-12}$ this technique has not gained widespread use. We report significantly better results using adjustable sutures compared with non-adjustable sutures, and suggest their use particularly for thyroid related upper eyelid retraction.

\section{Patients and methods}

The medical records of patients surgically treated for eyelid retraction at Moorfields Eye Hospital between January 1980 and October 1993 were reviewed. Patients were included in the study if there were two or more consistent preoperative measurements of the palpebral aperture in primary position and one of the following: marginal reflex distance (MRD, measurement of the distance from the corneal light reflex to the upper eyelid in primary position), an accurate drawing/photograph or documentation of millimetres of superior cornea covered by the upper eyelid. Values for the same measurements were required at postoperative day 2 , week 1 , week 6 , month 3 , between 3 and 6 months, and longest follow up. All patients with thyroid ophthalmopathy had stable eye disease, including exophthalmometer readings, for at least 6 months before their eyelid surgery, and during the follow up period. The outcome of surgery was evaluated when a stable palpebral aperture was achieved, defined as three consecutive readings all within $1.5 \mathrm{~mm}$ at three independent follow up visits. Surgical correction of the eyelid retraction was defined as good if (1) documentation existed that the eyelid covered between 1.0 to $3.0 \mathrm{~mm}$ of superior cornea, or the MRD was between 2 and $4 ;(2)$ the palpebral aperture was within $1 \mathrm{~mm}$ of the normal side (unilateral cases) or the second involved eye (bilateral cases); and (3) the eyelid margin contour was smooth. An acceptable result was designated if there was an asymmetry of (1) 1-2 mm between the eyelids, (2) no further surgery was required, and (3) the eyelid margin contour was smooth.

The methods we used for adjustable levator recession using both the anterior and posterior approach have been described in detail in a recent publication. ${ }^{12}$ The technique used for non-adjustable surgeries was similar with the exception of the lack of an adjustable suture. For adjustable surgery, $5 / 0$ or $6 / 0$ polyglactin sutures ( 11 eyelids) or $5 / 0$ or $6 / 0$ polyglycolic acid sutures (two eyelids) were used. For nonadjustable surgery, no sutures were used with the posterior approach; where sutures were placed in the anterior approach $6 / 0$ polyglactin or polyglycolic acid sutures were used (six eyelids). All sutures were adjusted the next day using topical anaesthesia. The details of the adjustment technique have been described by Collin and O'Donnell. ${ }^{12}$ Adjustments were made where necessary to achieve the desired height and/or contour. The MRD was set between 1.5 and $2.5 \mathrm{~mm}$ in the presence of severe postoperative swelling, and between 2.5 and $3.5 \mathrm{~mm}$ when only mild to moderate swelling existed. Adjustments were also made in the presence of good eyelid height to obtain smooth contour of each eyelid and eliminate lateral flare. Statistical comparisons were carried out using $\chi^{2}$ analysis and Fisher's exact tests.

\section{Results}

Adjustable sutures were used for the repair of eyelid retraction in 13 eyelids of 10 patients (seven females and three males) ranging in age between 19 and 71 years, with a mean of 48 years. Good results were obtained in 10 of the 13 eyelids $(77 \%)$. Non-adjustable sutures were used in 148 eyelids of 108 patients ( 85 females 
Table 1 Surgical treatment of eyelid retraction with good results ${ }^{\star}$

\begin{tabular}{|c|c|c|c|c|c|}
\hline & \multirow{2}{*}{$\begin{array}{l}\text { Total } \\
\text { no of } \\
\text { eyelids }\end{array}$} & \multicolumn{2}{|l|}{ Non-thyroid } & \multicolumn{2}{|l|}{ Thyroid } \\
\hline & & $\begin{array}{l}\text { Previous eyelid } \\
\text { surgery }\end{array}$ & $\begin{array}{l}\text { No previous } \\
\text { eyelid surgery }\end{array}$ & $\begin{array}{l}\text { Previous eyelid } \\
\text { surgery }\end{array}$ & $\begin{array}{l}\text { No previous } \\
\text { eyelid surgery }\end{array}$ \\
\hline $\begin{array}{l}\text { Adjustable sutures } \\
\text { Non-adjustable sutures }\end{array}$ & $\begin{array}{r}13 \\
148\end{array}$ & $\begin{array}{r}4 / 4(100 \%) \\
16 / 37(43 \%)\end{array}$ & $\overline{10 / 18}(56 \%)$ & $\begin{array}{l}3 / 5(60 \%) \\
7 / 22(32 \%)\end{array}$ & $\begin{array}{c}3 / 4(75 \%) \\
24 / 71(34 \%)\end{array}$ \\
\hline
\end{tabular}

${ }^{\star}$ Good result is defined in the Patients and method section.

and 23 males) ranging in age between 5 and 78 years, with a mean age of 42 years. A good result was obtained in 56 of the 148 eyelids $(39 \%)$. The mean preoperative palpebral aperture of all eyelids was 12.4 (SD 2.9) mm with a median of $12.0 \mathrm{~mm}$ and postoperatively $9.2(2.5) \mathrm{mm}$ with a median of $9.0 \mathrm{~mm}$. In the patients with unilateral retraction, the mean increased eyelid height compared with the normal side was $2.6(1.5) \mathrm{mm}$ preoperatively and $-0.5(0.32) \mathrm{mm}$ postoperatively. The mean preoperative eyelid retraction was comparable in both the non-adjustable and adjustable suture groups. The time to eyelid height stabilisation postoperatively was between 7 and 180 days (mean 30 days; median 21 days). Sixty six per cent of eyelids achieved a stable height within 1 month postoperatively. Significant postoperative swelling did not affect the time to stabilisation. There was no difference in time to stabilisation between adjustable and non-adjustable methods. Fifty one of the 161 eyelids (32\%) required further surgery, $38(74 \%)$ to further lower the eyelid and in $13(26 \%)$ to raise the eyelid. The overall mean preoperative levator function was $13.5(3.4) \mathrm{mm}$ and postoperatively $12 \cdot 7(3 \cdot 4) \mathrm{mm}$.

Adjustable and non-adjustable suture techniques were further analysed within two groups of patients: those with eyelid retraction from thyroid disease, and those with eyelid retraction from all other causes. Table 1 summarises the achievement of good results using adjustable and non-adjustable sutures.

\section{THYROID EYELID RETRACTION}

\section{Adjustable sutures}

Using adjustable sutures, good results were obtained in $67 \%(6 / 9$ eyelids in seven patients). Good results were obtained in $3 / 4$ $(75 \%)$ eyelids that had previously undergone surgery, as compared with $3 / 5(60 \%)$ eyelids that had not.

\section{Non-adjustable sutures}

Non-adjustable sutures were used in 22 eyelids that had previously undergone surgery, and 71 eyelids that had not. Of the 22 eyelids of patients who had previously undergone eyelid surgery (four unilateral, nine bilateral; 21 posterior, one anterior), a good result was obtained in seven (32\%), and an adequate result in one $(5 \%)$. Of the remainder, four $(18 \%)$ required further surgery (three to lower and one to raise), and eight (36\%) with a good height but poor contour did not undergo further surgery. Two more eyelids (9\%) remained high but the patients refused further surgery.

Of the 71 eyelids in 43 patients without previous eyelid surgery (15 unilateral, 28 bilateral; 65 posterior, six anterior), good results were obtained in $24(34 \%)$ and an adequate result in five $(7 \%)$. Of the remainder, $31(44 \%)$ eyelids required further surgery (three of these more than one surgery), 23 to lower and eight to raise the lid. A good height but poor contour was obtained in a further 10 eyelids (14\%), and an inadequate height not requiring surgery in one eyelid $(1 \%)$.

\section{NON-THYROID EYELID RETRACTION}

\section{Adjustable sutures}

The aetiology of the eyelid retraction in nonthyroid patients treated with adjustable sutures was: following ptosis surgery (three), trauma (one). Using adjustable sutures, all four patients with non-thyroid eyelid retraction had good results (three anterior approach, one posterior approach). None of the patients had significant swelling or required postoperative eyelid massage.

\section{Non-adjustable sutures}

The aetiologies of the eyelid retraction in nonthyroid patients treated with non-adjustable sutures were: congenital (seven), following ptosis (27), following other eyelid surgery (eyelid retraction and blepharoplasty) (nine), and trauma (12). Non-adjustable sutures were used in 37 eyelids that had previously undergone eyelid surgery, and 18 eyelids that had not. Of the 37 eyelids of patients who had previously undergone eyelid surgery ( 35 unilateral, one bilateral; 34 posterior, three anterior), a good result was obtained in $16(43 \%)$, and an adequate result in two $(5 \cdot 5 \%)$. Of the remainder, $10(27 \%)$ required further surgery (eight to lower the eyelid and two to raise it), and nine eyelids $(24 \cdot 5 \%)$ with good height but poor contour did not undergo further surgery. Of the 18 eyelids in 17 patients without previous eyelid surgery (16 unilateral, one bilateral; 17 posterior, one anterior), good results were obtained in $10(56 \%)$ and an adequate result in two $(11 \%)$. Of the remainder, six eyelids $(33 \%)$ required further surgery (four to lower and two to raise the eyelids).

\section{Discussion}

The surgical repair of upper eyelid retraction is significantly complicated by the inability to predict postoperative height and contour. In an attempt to obtain better results, numerous surgical procedures have been created involving surgery to weaken the levator aponeurosis and/or Mueller's muscle, and the use of various spacers. ${ }^{1-6} \mathrm{~A}$ range of postoperative results has been reported in the literature, but it is difficult to make comparisons between studies owing to differences in surgical techniques and variability in the definition of 
'success'. Putterman reported good results in $81 \%$ of 32 thyroid patients with levator recession and a graded excision of Mueller's muscle, ${ }^{13}$ although his defined criteria for a good result were poorly defined. Harvey and Anderson ${ }^{4}$ reported 16/24 (66\%) with good results using an anterior approach to recession of the levator aponeurosis combined with cutting of the lateral horn and extirpation of Mueller's muscle. However, the definition of 'desired postoperative height' is not given. Levine and Chu reported $87 \%$ success in 15 thyroid patients using a cutaneous approach to levator aponeurotic/Mueller's muscle recession $^{14}$; however, only three of their 15 patients $(20 \%)$ meet our criteria for a 'good' result. Applying strict criteria comparable with our own, Mourits and Koornneef reported only $50 \%$ 'good outcome' in patients with Graves' ophthalmopathy after one procedure using upper eyelid sclera. ${ }^{15}$ Hence, our results in patients treated with non-adjustable sutures are comparable with other studies where strict criteria for defining a good result were similarly applied. Our goal in this study was to evaluate our results using non-adjustable versus adjustable suture techniques in a group of patients undergoing uniform surgical approaches to the repair of upper eyelid retraction. The strict criteria we applied in our study were necessary to accurately compare our results using the adjustable and non-adjustable techniques. Although a greater proportion of patients were treated via a posterior approach in the non-adjustable suture category, our results remained unchanged when we analysed surgical outcomes using the anterior approach only: $7 / 10(70 \%)$ surgeries performed via an anterior approach with an adjustable suture technique had good results compared with $5 / 12(42 \%)$ using the non-adjustable technique. Although we found that small fluctuations in height can occur over a mean of 30 days, following the guidelines we described for the adjustment within 24 hours of surgery led to the significantly higher final 'good' results in this group compared with the non-adjustable surgical group.

Adjustable sutures are not new to eyelid surgery ${ }^{7-12}$; however, their use has not become popular for the treatment of eyelid retraction. Even with the small number of eyelids treated with the adjustable technique, good results were obtained significantly more often than for non-adjustable surgery $(77 \%$ versus $38 \%, \mathrm{p}<0.01)$. The time required for stabilisation of eyelid height postoperatively is not shortened by the use of adjustable sutures. We suggest the use of adjustable sutures in the repair of upper eyelid retraction especially for patients with thyroid disease, where results using non-adjustable sutures are particularly poor.

1 Thaller VT, Kaden K, Lane CM, Collin JRO. Thyroid lid surgery. Eye 1987; 1: 609-14.

2 Anderson JW. Relief of eyelid retraction. Arch Ophthalmol 1965; 74: 205-16.

3 Grove AS. Upper eyelid retraction: treatment by levator marginal myotomy. Orbit 1982; 1: 21-31.

4 Harvey JT, Anderson RL. The aponeurotic approach to eyelid retraction. Ophthalmology 1981; 88: 513-24.

5 Harvey JT, Corin S, Nixon D, Veloudios A. Modified levator aponeurosis recession for upper eyelid retraction in Graves' disease. Ophthalmic Surg 1991; 22: 313-7.

6 Older JJ. Surgical treatment of eyelid retraction associated with thyroid eye disease. Ophthalmic Surg 1991; 22 318-23.

7 Hylkema HA, Koornneef L. Treatment of ptosis by levator resection with adjustable sutures via the anterior approach. Br F Ophthalmol 1989; 73: 416-8.

8 Berris CE. Adjustable sutures for the correction of adult acquired ptosis. Ophthal Plast Reconstr Surg 1988; 4: acquired

9 Akyol N, Unucerci $\mathrm{CH}$. The correction of ptosis with adjustable suture technique. Acta Ophthalmol 1990 487-90.

10 Small RG. Controlled recession of the upper eyelid. Adv Ophthalmol Plast Reconstr Surg 1982; 1: 263-74.

11 Small RG. Upper eyelid retraction in Graves' ophthalmopathy. Trans Am Ophthalmol Soc 1988; 86: 725-93.

12 Collin JRO, O'Donnell BA. Adjustable sutures in eyelid surgery for ptosis and lid retraction. Br f Ophthalmol 1994, 78: $167-74$

13 Putterman AM. Surgical treatment of thyroid-related upper eyelid retraction. Graded Mueller's muscle excision and eyelid retraction. Graded Mueller's muscle excisio

14 Levine MR, Chu A. Surgical treatment of thyroid-related lid retraction: a new variation. Ophthalmic Surg 1991; 22: 90-4.

15 Mourits MP, Koornneef $L$. Lid lengthening by sclera interposition for eyelid retraction in Graves' ophthalmopathy. Br f Ophthalmol 1991; 75: 344-7. 